\title{
Composition Control of Low-Volatility Solids Through Chemical Vapor Transport Reactions. I. Theory of Selective Chemical Vapor Transport
}

\author{
A.Yu. Zavrazhnov, I.D. Zartsyn, A.V. Naumov, V.P. Zlomanov and A.V. Davydov
}

(Submitted June 29, 2006; in revised form June 3, 2007)

\begin{abstract}
A new method is proposed for controlling the composition (nonstoichiometry) of low-volatility inorganic compounds. It is based on the introduction/elimination of one of the components into/ from the low-volatility compound using reversible selective chemical vapor transport. The conditions for composition control through selective chemical vapor transport are deduced from the principles of nonequilibrium thermodynamics, in which the direction of the mass-transfer of a component is unambiguously defined by the temperature and composition of the source $\left(T_{1}, x_{1}\right)$ and of the sample $\left(T_{2}, x_{2}\right)$. This approach can be employed to control the sample composition in a closed vapor-transport system, in which composition $x_{2}$ of the sample is defined by the fixed values of $x_{1}, T_{1}$, and $T_{2}$, provided the steady-state (no mass-transfer) condition is achieved. The effect of steady-state conditions on sample composition is visualized using $T_{2}-T_{1}-x_{2}$ diagram for the following system: sample (Ga-S phases)-vapor-charge (pure Ga).
\end{abstract}

Keywords binary system, experimental kinetics, experimental techniques, experimental thermodynamics, modeling, nonequilibrium processes

\section{Introduction}

A chemical vapor-transport (CVT) method is commonly used for obtaining high-quality single crystals that are difficult or even impossible to grow by other methods. Instrumental difficulties emerge, for example, in the case of making compounds that melt at high temperature and high pressure. The CVT method was successfully applied for controlling composition of various inorganic compounds. ${ }^{[1-9]}$ However, composition of the crystals that are grown is strongly affected by many factors such as the nature and concentration of the gaseous species, temperature profile in the reactor and its geometry, composition of the source, presence of impurities, and so forth. As a result, it is very difficult to control the composition of a crystal that is grown by a CVT process.

In this work, the authors describe a new approach to control the composition of solids. The basic principle of this approach is the addition (or removal) of a component to a low-volatility crystal using selective chemical vaportransport (SCVT).

A.Yu. Zavrazhnov, I.D. Zartsyn, and A.V. Naumov, General Chemistry Department, Voronezh State University, Voronezh, Russia; V.P. Zlomanov, Inorganic Chemistry Department, Moscow State University, Moscow, Russia; A.V. Davydov, National Institute of Standards and Technology, 100 Bureau Drive, Stop 8554, Gaithersburg, MD 20899, USA. Contact e-mail: davydov@nist.gov.

The SCVT method has a number of features in common both with conventional annealing of solids in vapor of a volatile component ${ }^{[10]}$ and with classic CVT. ${ }^{[11]}$ The main difference from the classic CVT is that the sample composition still varies during the SCVT process, but the sample resublimation is prevented. In contrast to the conventional annealing, the system contains an additional substance capable of transporting one of the components from the source to the sink or in the opposite direction. Given the marked difference between the proposed and earlier approaches to compositional control, particular attention should be paid to thermodynamic analysis of the processes involved in SCVT method. The transport direction and the final steady-state conditions of the generally nonisothermal system must then be identified in a more complex manner compared with the known two-zone annealing processes. ${ }^{[10]}$ New experimental data, which support the concept of the SCVT method, have recently been discussed. ${ }^{[12]}$ This paper presents the theoretical analysis of this method.

SCVT method is based on reversible selective interaction of the component $A$ with a vapor-transport agent $A_{p} C_{q}$ $(p \geq 0, q \geq 1)$ :

$(n q-m p) A^{\prime \prime}+m A_{p} C_{q}^{\prime}=q A_{n} C_{m}^{\prime}$

where prime (') and double prime (") indicate vapor and condensed phases, respectively, and symbols $n, m, p$, and $q$ are the stoichiometric coefficients of the gaseous species $A_{p} C_{q}$ and $A_{n} C_{m}$. (For example, for the estimated reaction of gaseous $\mathrm{In}_{2} \mathrm{Cl}_{6}$ with condensed indium, which results in gaseous $\operatorname{In}_{2} \mathrm{Cl}_{4}$, one has $p=2, q=6, n=2$ and $m=4$. According to Eq 1, (2.6-4.2) $\mathrm{In}^{\prime \prime}+4 \mathrm{In}_{2} \mathrm{Cl}_{6}^{\prime}=6 \mathrm{In}_{2}$ $\mathrm{Cl}_{6}^{\prime}$ or $2 \mathrm{In}^{\prime \prime}+2 \mathrm{In}_{2} \mathrm{Cl}_{6}^{\prime}=3 \mathrm{In}_{2} \mathrm{Cl}_{4}^{\prime}$.

Analysis of the problem and experimental facts show that this reaction can be used to control composition of 


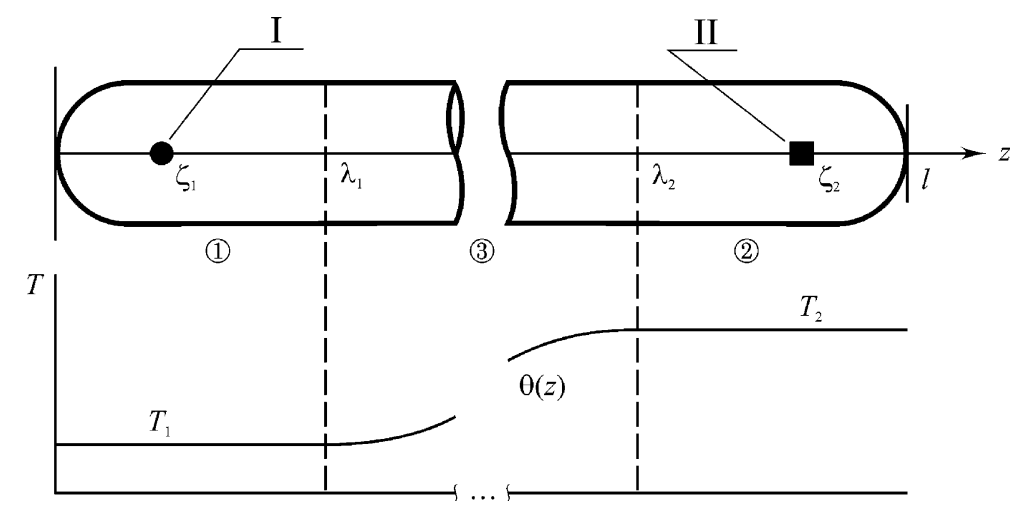

Fig. 1 Experiment for controlling composition of the sample $A_{x} B_{y}$ by using selective chemical vapor transport (with the corresponding temperature profile). I indicates the charge, which serves as source or getter of the component to be transported; II indicates the $A_{x} B_{y}$ sample

low-volatility compound $A_{x} B_{y}$, provided the following conditions are realized:

1. Interaction of transporting component $C$ with only one of the components in the $A_{x} B_{y}$ compound (e.g., component $A$ ) should: (a) supply comparable amounts of the forms $A_{p} B_{q}$ and $A_{n} C_{m}$ to the vapor phase and (b) quickly attain the equilibrium state.

2. No binary or ternary compounds of component $C$ with component $B$ (which will not be transported) should exist in the ternary $A-B-C$ system under experimental conditions (so-called "indifference condition for component $C$ in respect to the component $B$ ").

3. Component $C$ must be practically insoluble in $A_{x} B_{y}$ compound.

4. $A_{x} B_{y}$ compound must be nonvolatile at the experimental conditions.

When these requirements are met, the SCVT method can be used for controlling composition of $A_{x} B_{y}$ phase via annealing this compound in the atmosphere of transporting agent $C$ (in its certain forms, $A_{p} C_{q}, A_{n} C_{m}$ ) and in the presence of the charge - a condensed source or getter of component $A .^{[13-15]}$

\section{Control of the Composition for Low-Volatility Gallium Selenides}

In earlier work, ${ }^{[16,17]}$ the authors demonstrated the possibility of application of iodide transport to control the composition of low-volatility gallium selenides in the temperature range of $800 \leq T \leq 1000 \mathrm{~K}$ and pressure range of $3 \times 10^{4} \leq P \leq 3 \times 10^{5} \mathrm{~Pa}$, that is, at the conditions when ternary compounds (for example, $\mathrm{GaSe}^{[18]}$ ) are not stable. To control composition of gallium selenides, the following nonisothermal closed system was used: ${ }^{[12]} \mathrm{Sam}-$ ple at $T_{2}\left(\mathrm{GaSe}\right.$ or $\mathrm{Ga}_{2} \mathrm{Se}_{3}$ single crystals)-Vapor $\mathrm{GaI}$ + $\mathrm{GaI}_{3}$-Charge at $T_{1}$ (pure Ga) (quartz ampoule in the twozone horizontal furnace as shown in Fig. 1). Gallium tri-iodide $\left(\mathrm{GaI}_{3}\right)$ was applied as the initial transport agent. In this case, chemical transport is based on selective and reversible reaction

$2 \mathrm{Ga}^{\prime \prime}+\mathrm{IGaI}_{3}^{\prime}=3 \mathrm{GaI}^{\prime}$

In Eq 2, double prime (") indicates gallium from condensed gallium selenides $\left(\mathrm{GaSe}, \mathrm{Ga}_{2} \mathrm{Se}_{3}\right)$ or from a liquid gallium and prime (') stands for the gas species $\left(\mathrm{GaI}, \mathrm{GaI}_{3}\right)$. Since chemical potential for the component of ideal gas mixture can be represented as

$\mu_{i}=\mu_{i}^{\circ}(T)+R T \ln p_{i}$

where $i=\mathrm{GaI}, \mathrm{GaI}_{3} \cdot \mu_{i}{ }^{\circ}$ is the chemical potential under standard pressure for the pure component, and $p_{i}$ is the partial pressure of the same component; the necessary equilibrium condition (Eq 2) provides:

$R T \ln \kappa_{p}=2 \mu_{\mathrm{Ga}}^{\prime \prime}+F^{\prime}(T)$

where

$\kappa_{p}=p_{\mathrm{Gal}}^{3} / p_{\mathrm{Gal}_{3}}$

and

$F^{\prime}(T)=\mu_{\mathrm{Gal}_{3}}^{\circ}-3 \mu_{\mathrm{Gal}}^{\circ}$

where $F^{\prime}(T)$ term only depends on temperature.

In the gravimetric experiments (high-temperature gasphase balance ${ }^{[9]}$ ) in the aforementioned nonisothermal system, it was found that for certain compositions of sample and getter (gallium source) the direction of gallium transfer is only determined by the two values, the charge temperature $\left(T_{1}\right)$ and the sample temperature $\left(T_{2}\right) \cdot{ }^{[12]}$ Transfer of the component is continued until the stationary state is achieved with the zero flow of gallium $\left(J_{\mathrm{Ga}}\right)$, and both the sample and the getter reach thermodynamic equilibrium with the vapor phase.

It was experimentally shown that at the stationary state

$\left(\kappa_{P}\right)_{1}=\left(\kappa_{P}\right)_{2}$

where $\left(\kappa_{P}\right)_{1}$ and $\left(\kappa_{P}\right)_{2}$ stand for the $\kappa_{P}$ values for the charge and for the sample, correspondingly; $\left(\kappa_{P}\right)_{2}=f\left(T_{2}, x_{2}\right)$ and 
$\left(\kappa_{P}\right)_{1}=f\left(T_{1}, x_{1}\right)$ (in cases using pure component in the charge, $\left.\left(\kappa_{P}\right)_{1}=f\left(T_{1}\right)\right)$.

Here the authors also take into account that according to experimental data for a wide variety of systems, the pressure effect on the chemical potentials of components in solids and, hence, on $\kappa_{P}$ is negligible.

Following the empirical law (Eq 7) allows solving the inverse problem: determining compositions of the sample and of the getter that coexist in nonisothermal system at given $T_{1}$ and $T_{2}$, thus resulting in a precise compositional control of the gallium selenide phases by SCVT. However, previous works did not provide appropriate theoretical foundation for the SCVT method. Hence, the goal of this paper is to describe simultaneously chemical vapor-transport processes in a nonisothermal system.

\section{Stationary State in a Transport Nonisothermal System}

The simplest description is assumed for a system where there exists a single correlation of the type (Eq 1). Nonequilibrium processes in the system can be analyzed from the most general viewpoint without the use of ideas on the mechanism of transfer. The transfer mechanism is determined by the nature and composition of the gas phase; therefore, the equations describing the transport system should not contain the values referred to the gas phase (transport agents). One assumes that a system rather quickly attains constant pressure and considers it an approximation.

One can accept the following model. There are three subsystems (Fig. 1): two heterogeneous isothermal subsystems 1 and 2 and one homogeneous (vapor phase) subsystem 3 with continuously changing temperature field. In subsystem 2 one has the condensed sample to be controlled and in subsystem 1 one has the condensed charge, which acts as a source or a getter of the component to be transported.

This model corresponds to the experimental conditions in which the temperature field (furnace temperature profile) is described by the formula:

$T(z)=\left\{\begin{array}{l}T_{1}, 0 \leq z \leq \lambda_{1} \\ \theta(z), \lambda_{1} \leq z \leq \lambda_{2} ; \quad \theta\left(\lambda_{1}\right)=T_{1}, \theta\left(\lambda_{2}\right)=T_{2} \\ T_{2}, \lambda_{2} \leq z \leq l\end{array}\right.$

where $\theta(z)$ is a continuous, monotonically increasing function; $\lambda_{1}$ and $\left(l-\lambda_{2}\right)$ are the lengths of subsystems 1 and 2 , correspondently (Fig. 1). In case of usual transport experiment in a closed system, ${ }^{[11]}$ a one-dimensional model proves to be quite natural since it involves a single coordinate $z$. Its extension for a three-dimensional case does not result in any significant improvement of the model. As a rule, dimensions of condensed solids are much less than lengths $\lambda_{1}$ and $\left(l-\lambda_{2}\right)$, and therefore it is convenient to consider them as point bodies and to set their positions with coordinates $\zeta_{1}$ and $\zeta_{2}$, satisfying certain conditions in accordance with accepted stipulation, where:
$0 \leq \zeta_{1}<\lambda_{1}, \quad \lambda_{2}<\zeta_{1} \leq l$

In each of the isothermal subsystems 1 and 2 the chemical reaction $(\mathrm{Eq} 1)$ is a sole current process. It is known that production of entropy of chemical reaction can be described as: ${ }^{[19]}$

$\dot{S}=\frac{1}{T} A \mathrm{v}$

where $A=-\sum_{i} v_{i} \mu_{i}$ is chemical affinity; $\mathrm{v}=-\dot{n}_{i} / v_{i}$ is reaction rate; $i$ takes the values of $\mathrm{Ga}, \mathrm{GaI}, \mathrm{GaI}_{3}$. Since both sample and a source are assumed to be point objects, then local entropy production in subsystems 1 and 2 can be expressed as:

$\dot{s}_{1}=\frac{1}{T} A \mathrm{v} \delta\left(\zeta_{1}-z\right) \quad$ and $\quad \dot{s}_{2}=\frac{1}{T} A \mathrm{v} \delta\left(\zeta_{2}-z\right)$

where $\delta\left(\zeta_{\gamma}-z\right), \gamma=1,2$ is "shifted" Dirac function, and

$$
s(z)=\lim _{V \rightarrow P(z)} \frac{S(V)}{V}
$$

is density of entropy in point $P(z)$.

The partial pressures of saturated $\mathrm{Ga}$ and Se vapors (as well as of $\mathrm{Ga}_{2}$ Se species) over solid gallium selenides are by several orders of magnitude smaller than the pressure of gallium iodides; ${ }^{[16]}$ therefore, the presence of $\mathrm{Ga}$, Se, and $\mathrm{Ga}_{2} \mathrm{Se}$ species in the vapor can be neglected, and the gas phase consists of two components, GaI and $\mathrm{GaI}_{3}$. Since, according to the assumption mechanical equilibrium is set over the whole system $\left(\nabla p=0^{[20]}\right)$, diffusion and heat transfer in subsystem 3 are irreversible current processes. In this case, the expression for local entropy production transforms into: ${ }^{[20]}$

$$
\dot{s}_{3}=\mathbf{J}_{U} \nabla\left(\frac{1}{T}\right)-\sum_{k}{ }_{k=\mathrm{GaI}, \mathrm{GaI}_{3}} \mathbf{J}_{k} \nabla\left(\frac{\mu_{k}}{T}\right)
$$

where $\mathbf{J}_{U}$ is the energy flow; and $\mathbf{J}_{k}$ are diffusion flows. The latter equation can be easily transformed to

$\dot{s}_{3}=\left(\mathbf{J}_{U}-\sum_{k} \mathbf{J}_{k} \mu_{k}\right) \nabla\left(\frac{1}{T}\right)-\frac{1}{T} \sum_{k} \mathbf{J}_{k} \nabla \mu_{k}$

Note, however, thermodynamic driving forces determining transfer of energy and substance are not yet separated there. Taking into account that chemical potential $\mu_{k}$ is a function of $T$ and $p_{k}$, one can write:

$\nabla \mu_{k}=\frac{\partial \mu_{k}}{\partial T} \cdot \nabla T+\left(\nabla \mu_{k}\right)_{T}$

where $\left(\nabla \mu_{k}\right)_{T}$ is a gradient of potential stipulated only by the change of vapor phase composition. Then the required separation of the generalized forces can be performed using "reduced heat flow"[19]

$\mathbf{J}_{Q}=\mathbf{J}_{U}-\sum_{k} \mathbf{J}_{k} H_{k}$

where $H_{k}$ is partial molar enthalpy. Equation (13) then takes the form of: 
$\dot{s}_{3}=\mathbf{J}_{Q} \nabla\left(\frac{1}{T}\right)-\frac{1}{T} \sum_{k} \mathbf{J}_{k}\left(\nabla \mu_{k}\right)_{T}$

One can proceed to the independent scalar fields in Eq 16 using Gibbs-Duhem equation:

$C_{\mathrm{GaI}}\left(\nabla \mu_{\mathrm{GaI}}\right)_{T}+C_{\mathrm{GaI}_{3}}\left(\nabla \mu_{\mathrm{GaI}_{3}}\right)_{T}=0$

where $C_{i}$ is the density of a substance (local concentration) and condition of the absence of the volume flow at $\nabla p=0$ :

$\mathbf{J}_{\mathrm{GaI}} V_{\mathrm{GaI}}+\mathbf{J}_{\mathrm{GaI}_{3}} V_{\mathrm{GaI}_{3}}=0$

where $V_{k}$ is a partial molar volume. It is clear that the latter condition in the case of ideal gases gives $\mathbf{J}_{\mathrm{Gal}}=-\mathbf{J}_{\mathrm{Gal}_{3}}$. Equation 16 finally transforms into:

$\dot{s}_{3}=\mathbf{J}_{Q} \nabla\left(\frac{1}{T}\right)-\frac{1}{T} \frac{\mathbf{J}_{\mathrm{GaI}}}{\left(1-x_{\mathrm{GaI}}\right)}\left(\nabla \mu_{\mathrm{GaI}}\right)_{T}$

Combining Eq 11 and 19, for local entropy production in each point of transport system $\dot{s}=\dot{s}_{1}+\dot{s}_{2}+\dot{s}_{3}$ gives:

$$
\begin{gathered}
\dot{s}=\mathbf{J}_{Q} \nabla\left(\frac{1}{T}\right)-\frac{1}{T} \frac{\mathbf{J}_{\mathrm{GaI}}}{\left(1-x_{\mathrm{GaI}}\right)}\left(\nabla \mu_{\mathrm{GaI}}\right)_{T} \\
+\frac{1}{T} A \mathrm{v} \sum_{\gamma} \delta\left(\zeta_{\gamma}-z\right) \\
\gamma=1,2
\end{gathered}
$$

In fact, the number of terms containing $\delta\left(\zeta_{\gamma}-z\right)$ can be more than two that correspond to the existence of several condensed samples. In this case, the isothermal condition must be satisfied: the temperature field must be homogeneous in a certain vicinity of the point $\zeta_{\gamma}$.

General entropy production over the entire closed transport system

$\dot{S}=\int_{V} \dot{s} d V$

is expressed by

$\dot{S}=\int_{0}^{l}\left(\mathbf{J}_{Q} \nabla\left(\frac{1}{T}\right)-\frac{1}{T} \frac{\mathbf{J}_{\mathrm{GaI}}}{\left(1-x_{\mathrm{GaI}}\right)}\left(\nabla \mu_{\mathrm{GaI}}\right)_{T}\right) d z \cdot \sigma+\sum_{\gamma} \frac{1}{T_{\gamma}} A_{\gamma} \mathrm{v}_{\gamma}$

(where $\sigma$ is a cross-sectional area). Note that Eq 22 includes the terms of two kinds that are different by their tensor dimensionality. One of these terms contains flows and forces that are vector values, while the other one includes only scalar values. Since the system is isotropic, evolution of the components that have vector and scalar nature in production of entropy with time should be considered separately. One should also note that the terms of the first kind are related only with subsystem 3 , while the other terms are related with subsystems 1 and 2 .

Consider a component

$$
\dot{S}^{\chi}=\sum_{\gamma} \frac{1}{T_{\gamma}} A_{\gamma} \mathrm{v}_{\gamma}
$$

of the total production connected with chemical reaction. At the boundary conditions (in this case, these are the values of temperature $T_{1}$ and $T_{2}$ ) inequality is true at any moment ${ }^{[19]}$

$\mathrm{v}_{1} d A_{1}+\mathrm{v}_{2} d A_{2} \leq 0$

and a sign of strict equality is related with a stationary state. Since reactions at the points $\zeta_{1}$ and $\zeta_{2}$ are independent, the conditions for stationary state are:

$A_{1}=$ const,$\quad A_{2}=$ const,$\quad$ or $\quad \mathrm{v}_{1}=0, \quad \mathrm{v}_{2}=0$

Boundary conditions applied to the system are not connected with the values of $A_{1}$ and $A_{2}$. In the closed system, the reaction in $\mathrm{Eq} 1$ can result in the change of composition of solid phases and, therefore, in the change of the values $A_{1}$ and $A_{2}$. Then any non-zero values of $A_{1}$ and $A_{2}$ cannot remain constant in the general case. An exceptional case is just the situation when one of the phases is completely consumed during the process of irreversible reaction. This case will not be further considered. Thus, a single constant value of chemical affinity in the stationary state is equal to zero:

$A_{1}=0, \quad A_{2}=0, \quad \mathrm{v}_{1}=0, \quad \mathrm{v}_{2}=0$

Considering the contribution of vector values into the entropy production one can neglect thermodiffusion phenomena in gas phase. In this case, it is natural to assume that they are in the range of linear kinetic relations (correlations between the flows $\mathbf{J}_{k}$ and forces $\mathbf{X}_{k}$ ):

$\mathbf{J}_{k}=L_{k} \mathbf{X}_{k}$

Then, under predetermined values of temperature $T_{1}, T_{2}$, the functional

$\dot{S}_{3}=\int_{V}\left(\mathbf{J}_{Q} \nabla\left(\frac{1}{T}\right)-\frac{1}{T} \frac{\mathbf{J}_{\mathrm{GaI}}}{\left(1-x_{\mathrm{GaI}}\right)}\left(\nabla \mu_{\mathrm{GaI}}\right)_{T}\right) d V$

will take its minimum value at condition: ${ }^{[19]}$

$\left(\nabla \mu_{\mathrm{GaI}}\right)_{T}=0$

(in accordance with the theorem of the minimum in entropy production).

According to Gibbs-Duhem equation the same condition should be realized for $\mathrm{GaI}_{3}$ :

$\left(\nabla \mu_{\mathrm{GaI}_{3}}\right)_{T}=0$

Thus, in a stationary state the following equalities are true:

$\nabla p_{\mathrm{GaI}}=0, \quad \nabla p_{\mathrm{GaI}_{3}}=0$

or

$p_{\mathrm{GaI}}\left(T_{1}\right)=p_{\mathrm{GaI}}\left(T_{2}\right), \quad p_{\mathrm{GaI}_{3}}\left(T_{1}\right)=p_{\mathrm{GaI}_{3}}\left(T_{2}\right)$

Combining conditions of Eq 26 and 32 with Eq 5 for the value of $\kappa_{p}$, one finds that the compositions of condensed phases (sample and getter) tend toward a stationary condition of the transport system in such a way that the condition in Eq 7 is satisfied: $\left[\left(\kappa_{P}\right)_{1}=\left(\kappa_{P}\right)_{2}\right.$; where 
Table 1 The temperature dependence of $\kappa_{P}$ values for different equilibrium states in the Ga-S-I system and the boundary lines that limit areas of existence of different condensed phases at $T_{2}-T_{1}$ projection of $T_{2}-T_{1}-x_{2}$ diagram. In column 2, prime (') and double prime (") indicate vapor and condensed phases, respectively

\begin{tabular}{|c|c|c|c|c|}
\hline 1 & 2 & 3 & 4 & 5 \\
\hline $\begin{array}{l}\text { Equilibrium phases } \\
\left(\mathrm{V}=\text { vapor } \mathrm{GaI}+\mathrm{GaI}_{3}\right)\end{array}$ & $\begin{array}{l}\text { Equilibrium in a } \\
\text { chemical subscription }\end{array}$ & $\begin{array}{l}\text { Temperature } \\
(T), \mathrm{K}\end{array}$ & $\begin{array}{l}\text { Temperature } \\
\text { dependence of } \kappa_{P}[16,17]\end{array}$ & $\begin{array}{l}\text { Temperature dependence of } T_{1}=f\left(T_{2}\right) \text {. } \\
\text { Calculated using Eq } 7 \text { and } \\
\text { data of the column } 4\end{array}$ \\
\hline $\mathrm{Ga}^{\mathrm{L}}-\mathrm{V}$ & $2 \mathrm{Ga}^{l}+\mathrm{GaI}_{3}^{\prime}=3 \mathrm{GaI}^{\prime}$ & $727-1134$ & $\log \kappa_{P}=\frac{-10,329}{T}+11.71$ & $T_{1}=T_{2}$ \\
\hline $\mathrm{L}^{\mathrm{Ga}}-\mathrm{GaS}^{\mathrm{s}}-\mathrm{V}$ & $\mathrm{Ga}^{\prime \prime}+\mathrm{GaI}_{3}^{\prime}=3 \mathrm{GaI}^{\prime}$ & $804-1065$ & $\log \kappa_{P}=\frac{-11,172}{T}+12.45$ & $T_{1}=\frac{10,329 T_{2}}{11,172-0.74 T_{2}}$ \\
\hline $\mathrm{GaS}^{\mathrm{s}}-\mathrm{Ga}_{2} \mathrm{~S}_{3}^{\mathrm{s}}-\mathrm{V}$ & $6 \mathrm{GaS}^{\mathrm{s}}+\mathrm{GaI}_{3}^{\prime}=3 \mathrm{GaI}^{\prime}+2 \mathrm{Ga}_{2} \mathrm{~S}_{3}^{\mathrm{s}}$ & $873-1130$ & $\log \kappa_{P}=\frac{-20,475}{T}+19.48$ & $T_{1}=\frac{10,329 T_{2}}{20,475-7.77 T_{2}}$ \\
\hline
\end{tabular}

$\left(\kappa_{P}\right)_{2}=f\left(T_{2}, x_{2}\right)$ and $\left(\kappa_{P}\right)_{1}=f\left(T_{1}, x_{1}\right)$; in case of pure component in the charge $\left.\left(\kappa_{P}\right)_{1}=f\left(T_{1}\right)\right]$.

The state when the condition in Eq 7 is satisfied corresponds to the minimum of entropy production, and now entropy production can be considered as dissipation potential, which attains its minimum value in a stationary state. This stationary state is a single one for each pair of values $T_{2}, T_{1}$. Moreover, the change of $T_{2}$ (at the specified value of $T_{1}$ ) or $T_{1}$ (at the specified value of $T_{2}$ ) will result in the new changes of sample composition (due to the loss or gain of some part of gallium) and in attaining a new stationary state that is different from the previous one but with invariable composition of a sample independent on the time.

Using Eq 4 to 6, the relationship in $\mathrm{Eq} 7$ can be written in the form of:

$\frac{2 \mu_{\mathrm{Ga}}^{\prime \prime}\left(T_{2}, x_{2}^{\prime \prime}\right)+F^{\prime}\left(T_{2}\right)}{T_{2}}=\frac{2 \mu_{\mathrm{Ga}}^{\prime \prime}\left(T_{1}, x_{1}^{\prime \prime}\right)+F^{\prime}\left(T_{1}\right)}{T_{1}}$

where subscripts 1 and 2 refer to the gallium source (getter) and the sample, respectively, and the quantities $F\left(T_{2}\right)$ and $F\left(T_{1}\right)$ are given above by $\mathrm{Eq} 6$. The relation in $\mathrm{Eq} 33$ is written according to the assumption of negligibly small pressure effect on the chemical potentials for solids. This relation shows that the sample composition $\left(x_{2}\right)$ in the stationary state is a function of three variable values: $T_{2}$ (temperature of a sample), $T_{1}$ (temperature of a source/ getter material), and $x_{1}$ (composition of a source/getter material).

In order to predict sample composition it is convenient to fix the composition of source/getter $\left(x_{1}\right)$, thereby eliminating one of the variables from consideration. Then the sample composition will be a function of only two variables $-T_{2}$ and $T_{1}$. This allows association of each vapor-phase transport system (with a certain transporting component and certain source/getter) that is in a stationary state and without any substance transfer with a three-dimensional $T_{2}-T_{1}-x_{2}$ diagram. Its physical meaning implies that the sample composition is self-adjusted to the selected temperatures $T_{2}$ and $T_{1}$. Taking into account the properties of chemical potentials and considering Eq 7, one can conclude that in the ranges of existence of the phases with variable compositions the following is true:
- Each nonstoichiometric composition of a sample $\left(x_{2}\right)$ is associated with strictly determined values $T_{2}$ and $T_{1}$.

- Under continuous change of $T_{2}$ or/and $T_{1}$ values, nonstoichiometric composition of a sample (at $T_{2}$ ) varies continuously as well.

The $T_{2}-T_{1}-x_{2}$ diagram for a stationary state of the vaportransport system has much in common with a classic equilibrium $P-T-x$ phase diagram.

To construct the $T_{2}-T_{1}-x_{2}$ diagram or its projections, the composition of a source/getter $\left(x_{1}\right)$ must remain invariable under evolution of the transport system to a stationary state. Invariability of the value $x_{1}$ can be attained by one of the three possible ways:

- Using a large amount of the charge relative to the sample amount

- Heterophase mixture made of two adjacent binary condensed phases (noteworthy, in a mixture of two coexisting phases, the chemical potential is fixed at a given temperature and does not depend on the overall composition of the mixture)

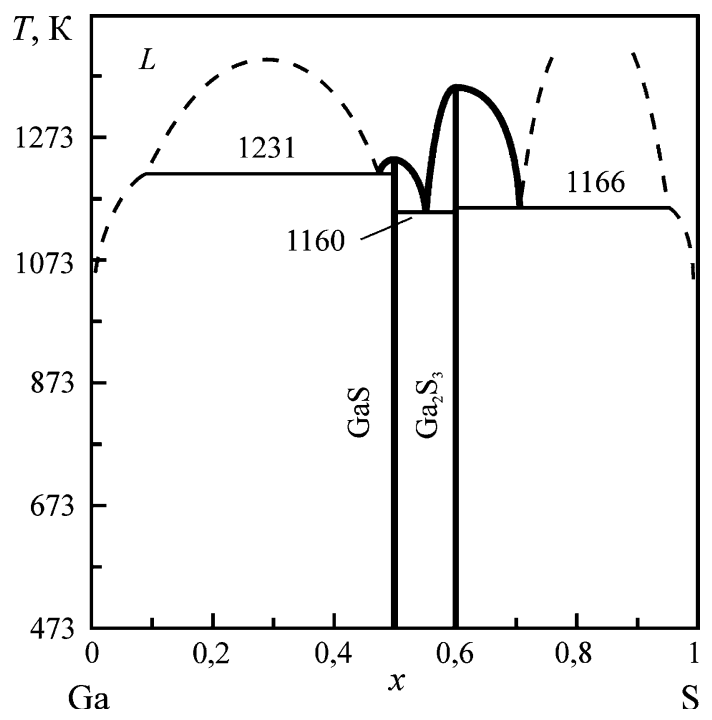

Fig. $2 T-x$ diagram of Ga-S system. Source: Ref 17 


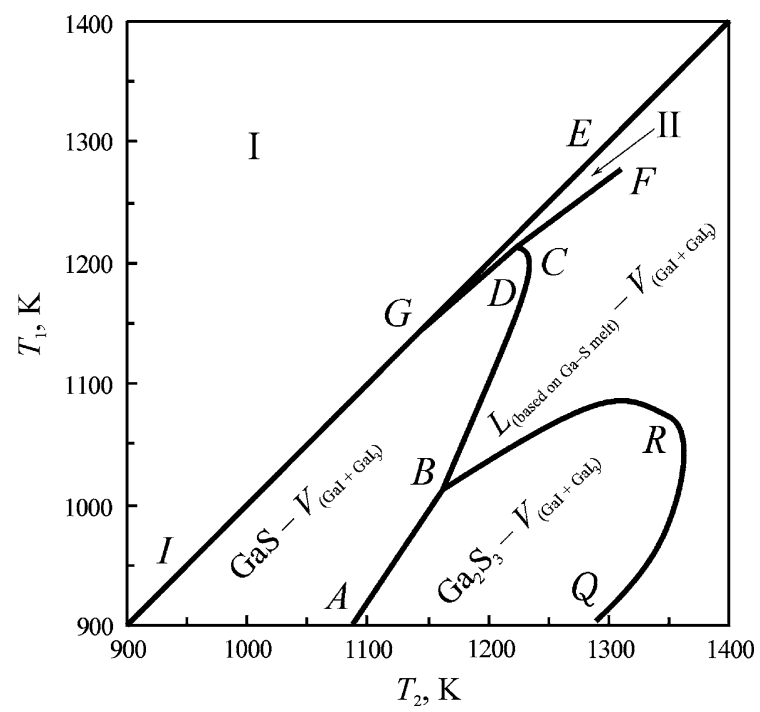

Fig. 3 Calculated boundary lines that limit areas of stability for phases in the Ga-S system. Lines of three-phase equilibria: $\mathrm{AB}-\mathrm{GaS}^{\mathrm{s}}-\mathrm{Ga}_{2} \mathrm{~S}_{3}{ }^{\mathrm{s}}-V ;$ BRQ $-L-\mathrm{Ga}_{2} \mathrm{~S}_{3}{ }^{\text {sol }}-V ;$ BCD- $L-\mathrm{GaS}^{\mathrm{sol}}-V$. Positions of the following lines are given tentatively: $\mathrm{IE}_{-} \mathrm{Ga}^{\text {liq. }}-V$; DF- $L$-Ga ${ }^{\text {liq., }}-V$; GD-Ga ${ }^{\text {liq., }}, \mathrm{GaS}^{\text {sol }}-V$, where $L$ is melt of variable composition $\mathrm{Ga}_{x} \mathrm{~S}_{1-x}, \mathrm{Ga}^{\text {liq. }}$ is melt of practically pure gallium (see also Fig. 2), and $V$ is vapor of $\mathrm{GaI}$ and $\mathrm{GaI}_{3}$. Field I experimentally unattainable field where $T_{1}>T_{2}$ (since $T_{2}$ should always be greater than $T_{1}$ ); Field II - area of co-existence of liquid (based on gallium) and vapor $\left(\mathrm{GaI}+\mathrm{GaI}_{3}\right)$ phases

- Using the pure component ( $A$ in Eq 1) as a source/getter material

The latter technique appears to be the most straightforward and convenient method to control the composition of gallium selenides and sulfides. In the recent study, ${ }^{[12]}$ it was shown what phases should form at a stationary state at different $T_{2}$ and $T_{1}$ values; if a sample of arbitrary composition from the Ga-Se system was chosen, pure metallic gallium was used as a source/getter and vapor phase was formed by gallium iodides. The $T_{2}-T_{1}$ projection for the Ga-Se $T_{2}-T_{1}-x_{2}$ diagram is given in Ref 12 .

In this follow-up work, the authors demonstrate similar results on $T_{2}-T_{1}$ projection of the $T_{2}-T_{1}-x_{2}$ diagram for $\mathrm{Ga}-\mathrm{S}$ phases, which would exist at a stationary state in the following vapor-transport system:

Sample ( $\mathrm{GaS}$ or $\mathrm{Ga}_{2} \mathrm{~S}_{3}$ single crystals) at $T_{2}$ - Vapor $\left(\mathrm{GaI}+\mathrm{GaI}_{3}\right)-$ Charge (pure Ga) at $T_{1}$.

Each condensed phase is plotted in this diagram as a field. The exact composition (nonstoichiometry) of this phase is established by the values of the $\left(T_{2}, T_{1}\right)$ pair. If the $\left(T_{2}, T_{1}\right)$ pair exceeds the bounds of a phase field, then the phase of the sample will transform into another phase. For example, when the difference between $T_{2}$ and $T_{1}$ is sufficiently high, the $\mathrm{GaS}$ phase will transform into $\mathrm{Ga}_{2} \mathrm{~S}_{3}$ (and the excess of gallium transfers back to the charge).

It is presumed that the assumptions used for the Ga-Se-I system also hold for the Ga-S-I system. In earlier studies, ${ }^{[16,17]}$ the values of $\kappa_{p}$ were determined for different three-phase equilibrium states (two binary condensed phases in the Ga-S system plus vapor phase made of gallium iodides) (Table 1). Based on Eq 7, it is possible to calculate $T_{2}-T_{1}$ projection of the $T_{2}-T_{1}-x_{2}$ diagram at a stationary state for the Ga-S-I gas-transport system. ${ }^{[12]}$ (Since the case of using metallic gallium as a source/getter was considered in the work, the expression from the first row of Table 1 was always taken to determine the temperature dependence of $\kappa_{p}\left(T_{1}\right)$ value.) The results of this calculation are given in Fig. 2 and 3. Selected tests using gravimetric technique (for details, see Ref 12), demonstrated a good agreement between predicted values and experimental data. For example, it was shown, that if the experimental differences between $T_{2}$ and $T_{1}$ exceed $\Delta T=180 \mathrm{~K}$ for $T_{1}=900 \mathrm{~K}$ (or $\Delta T=140 \mathrm{~K}$ for $T_{1}=1020 \mathrm{~K}$ ), the GaS sample transforms into $\mathrm{Ga}_{2} \mathrm{~S}_{3}$ (while the excess $\mathrm{Ga}$ transfers to the charge zone). On the contrary, when the $\mathrm{Ga}_{2} \mathrm{~S}_{3}$ sample is introduced into the SCVT system with the difference between $T_{2}$ and $T_{1}$ being smaller than $\Delta T<160 \mathrm{~K}$ for $T_{1}=900 \mathrm{~K}$ (or $\Delta T<120 \mathrm{~K}$ for $T_{1}=1020 \mathrm{~K}$ ), this sample absorbs gallium and transforms into GaS phase. These experimental data agree well with the calculation results, which yield for the $\mathrm{AB}$ boundary in Fig. 3 the value of $\Delta T=165 \mathrm{~K}$ at $T_{1}=900 \mathrm{~K}$ and $\Delta T=130 \mathrm{~K}$ at $T_{1}=1020 \mathrm{~K}$.

\section{Conclusions}

The conditions for composition control through selective chemical vapor transport (SCVT) are described from the principles of nonequilibrium thermodynamics. It is shown that the direction of mass transfer is unambiguously defined by the temperature $\left(T_{1}, T_{2}\right)$ and composition $\left(x_{1}, x_{2}\right)$ of the source and of the sample, respectively. This approach can be employed to control sample composition in a closed vaportransport system, in which composition $x_{2}$ of the sample is defined by the fixed values of $x_{1}, T_{1}$ and $T_{2}$ providing a steady state without mass transfer. The effect of steady-state conditions on sample composition could be visualized using $T_{2}-T_{1}-x_{2}$ diagram that has much in common with the classical $P-T-x$ diagram for equilibrium conditions.

\section{References}

1. F.H. Wehmeier, The Growth of Cadmium Chromium Selenide by Chemical Transport with Cadmium Chloride and the Behavior of Cadmium Chromium Selenide at Elevated Temperatures, J. Cryst. Growth, 1969, 5, p 26

2. P. Gibart and C. Vacherand, Preparation de Monocristaux de Tellurures de Cobalt, J. Cryst. Growth, 1969, 5, p 111 (in French)

3. M. Schäter, Chemical Transport Reactions in Gases and Some Remarks Concerning Melts and Fluid Phases, Chemistry and Geochemistry of Solutions at High Temperatures and Pressures. Proc. Nobel Symp. Karlskoga, Sept 1979, Phys. Chem. Earth, 1981, 13/14, p 409

4. S. Mong and O. Hy, Crystal Growth of Some Intermediate Titanium Oxygen Phases $\gamma-\mathrm{Ti}_{3} \mathrm{O}_{5}, \beta-\mathrm{Ti}_{3} \mathrm{O}_{5}, \mathrm{Ti}_{4} \mathrm{O}_{7}$, and $\mathrm{Ti}_{2} \mathrm{O}_{3}$ 
by Chemical Transport Reactions, Acta Chem. Scand., 1982, A36, p 207

5. F. Levy and H. Berger, Single Crystals of Transition Metal Trichalcogenides, J. Cryst. Growth, 1983, 61, p 61

6. E.E. Trifonora, L. Karagiozov, and L. Hitova, Application of the Iodine Synthesis to the Preparation of Indium Arsenide Bulk Crystals and Epitaxial Layers, Cryst. Res. Technol., 1983, 18, p 1341

7. M.H. Kimmel, M.-Ch. Lux-Steiner, M. Pbergfell, and E. Bucher, Growth and Characterization of Low Resistive $\mathrm{CdSiAs}_{2}$ Single Crystals, VIII. Int. Conf. Solid Compounds Transit. Elem. (Vienna, Austria), April 9-13, 1985; Extended Abstr., Vienna, 1985. PUA (2) -P4A (2)3

8. M. Lenz and R. Gruehn, Developments in Measuring and Calculating Chemical Transport Phenomena Demonstrated on Cr, Mo, W and Their Compounds, Chem. Rev., 1997, 97, p 2967

9. R. Gruehn and R. Glaum, New Results of Chemical Transport as a Method for the Preparation and Thermochemical Investigation of Solids, Angew. Chem. (Int. Ed.) 2000, 39, p 692-716

10. F.A. Kröger, Chemistry of Imperfect Crystals. Philips' Research Laboratory, North-Holland Publishing, Amsterdam, 1964, p 654

11. H. Schäfer, Chemische Transportreaktionen Der Transport Anorganischer Stoffe über die Gasphase und seine Anwendungen. Verlag Chemie, GmbH Weinheim/Bergstr, 1964 (in German)
12. A.Yu. Zavrazhnov, D.N. Turchen, and A.V. Naumov, Chemical Transport Reactions as a New Variant of the Phase Composition Control, J. Phase Equilibria, 2003, 24(4), p 330

13. H. Schaefer, R. Gruehn, and F. Schulte, Die Modifikationen des Niobpentoxids, Angew. Chem., 1966, 78(1), p 28 (in German)

14. H. Hibst, R. Gruehn, and D. Weitere, Die Neue $\mathrm{Nb}_{2} \mathrm{O}_{5^{-}}$ Modifikationen, Metastabile Oxidationsprodukte von NbOxPhasen $(2,4<x<2,5)$, Z. Anorg. Allg. Chem, Bd., 1978, 442, p 49 (in German)

15. H. Schaefer, D. Bergner, and R. Gruehn, Die Thermodynamische Stabilitat der Sieben Zwischen 2,00 und 2,50 O/Nb existierenden Phasen, Z. Anorg. Allg. Chem., 1969, 365, p 31

16. A. Zavrazhnov, D. Turchen, E. Goncharov, and V. Zlomanov, Manometric Method for the Study of P-T-X Diagrams, J. Phase Equilibria, 2001, 22(4), p 482

17. A.Yu. Zavrazhnov, P-T-x Phase Diagrams of Gallium Chalcogenides Studied Using an Additional Component, Russ. J. Inorg. Chem., 2003, 48(10), p 1722

18. R. Kniep, A. Wilms, and H.J. Beister, Phase Relation in Ga2X3-GaY3 System: Crystal Growth, Structural Relation and Optical Absorption of intermediate compounds GaXY, Mater. Res. Bull., 1983, 18, p 615

19. I. Prigogine, Introduction to Thermodynamics of Irreversible Processes. C.C. Thomas, Springfield, 1955

20. R. Haase, Thermodynamik der Irreversiblen Prozesse, Dr. D. Steinkopf Verl., Darmstadt, 1963 (in German) 